\title{
Congenital malformations and testicular germ cell tumors
}

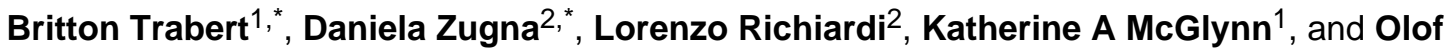 \\ Akre $^{2,3}$ \\ ${ }^{1}$ Hormonal and Reproductive Epidemiology Branch, Division of Cancer Epidemiology and \\ Genetics, National Cancer Institute, Rockville, MD \\ ${ }^{2}$ Cancer Epidemiology Unit, Department of Medical Sciences, University of Turin, and CPO- \\ Piemonte, Turin, Italy \\ ${ }^{3}$ Clinical Epidemiology Unit, Karolinska Institute, Stockholm, Sweden
}

\section{Abstract}

Cryptorchidism is one of the few known risk factors for testicular germ cell tumors (TGCT). It has been postulated that other congenital malformations, in particular hypospadias, are also associated with increased risk; however, associations with birth defects have not been extensively studied. Using Swedish population-based registries we evaluated the relationship between birth defects and risk of TGCT. TGCT cases $(n=6,593)$ diagnosed between 15 and 65 years of age were identified from the Swedish Cancer Registry between 1964 and 2008. Five controls per case were randomly selected from the population register and matched on birth year and birth county. Congenital malformations were identified via linkage with the Hospital Discharge Register. Odds ratios (OR) and $95 \%$ confidence intervals (CI) for the association between each group of malformations and TGCT were estimated using conditional logistic regression. In addition to the expected association between cryptorchidism and TGCT risk [OR (95\% CI): 3.18 (2.50 to 4.04)], hypospadias [2.41 (1.27 to 4.57)], inguinal hernia [1.37 (1.11-1.68)] and other genital malformations [2.19 (1.17 to 4.10)] were associated with an increased risk of TGCT. Mutual adjustment for cryptorchidism, hypospadias, inguinal hernia, and other genital malformations did not appreciably change the associations (ORs: $3.16 ; 2.25 ; 1.30 ; 1.90$, respectively). The other (non-genital) malformations evaluated were not associated with TGCT. These data suggest that developmental urogenital abnormalities, specifically cryptorchidism, hypospadias, and inguinal hernia, are associated with an increased risk of TGCT; further supporting the hypothesis that prenatal exposure(s) related to proper genital development are related to this tumor.

\section{Keywords}

congenital malformations; testicular germ cell tumors; hypospadias; cryptorchidism

Corresponding author: Britton Trabert, 6120 Executive Blvd, Rockville MD 20852-7234, Phone: 301-451-4435, Fax: 301-402-7234, britton.trabert@nih.gov.

* contributed equally 


\section{INTRODUCTION}

Testicular germ cell tumors (TGCT) are the most common neoplasm among young men aged 15-40 years in many parts of the world and the incidence has steadily increased over the past 50 years in many populations (1). A growing body of evidence suggests that TGCT may have a perinatal etiology (2). The etiologic role of congenital malformations and TGCT has focused on cryptorchidism and to a lesser extent hypospadias (3). A meta-analysis estimated the relative risk of TGCT among men with prior cryptorchidism to be 4.3 (95\% confidence interval (CI): 3.6-5.1)(4). A recent nation-wide study from Denmark estimated a 2.1 increased risk for testicular cancer in association with hypospadias, based on 14 exposed subjects (5). There are few data on the association between other birth defects and risk of TGCT, mainly due to the difficulty of studying rare exposures in combination with a rare tumor with an incidence of less than 10 per 100,000. We assessed the association of congenital malformations and TGCT risk in a Swedish population-based case-control study utilizing the Swedish Cancer Registry, the Medical Birth Register of Sweden, the Hospital Discharge Register, and the Register of Populations and Population Changes.

\section{MATERIAL AND METHODS}

\section{Data sources}

This study was conducted using population-based registry data from Sweden. Linkage of individual information on birth characteristics, hospitalizations, and cancer incidence was possible through a personal identity number (PIN) unique to every Swedish resident. Reporting of cancer cases is compulsory and the Swedish Cancer Registry covers the entire population from 1958. However because hospitalization records were not available prior to 1964, we selected all TGCT cases diagnosed between 15 and 65 years of age and registered in the Swedish Cancer Registry between 1964 to 2008 (ICD-7 178; ICD-8 \& ICD-9 186; ICD-10 C62).

The Swedish Medical Birth Register is a population-based registry that contains information on all births in Sweden since 1973. Through this registry we obtained birth characteristics for all cases and controls born after 1973. Hospitalizations from congenital malformations, including cryptorchdism and hypospadias, were obtained from the Hospital Discharge Register. The Hospital Discharge Register covered 6 out of 26 health care regions in Sweden in 1964 and increased coverage to 16 of 26 in 1975 to complete coverage in 1987. Provided that virtually all patients in Sweden are treated at public hospitals, the Hospital Discharge Register is representative of the hospitalizations in Sweden. To identify malformations we used the diagnostic codes (summarized in Supplemental Table 1) and, for cryptorchidism and hypospadias/epispadias we further used codes of surgical procedures to distinguish patients who underwent operation from those who did not. For all other (non-genital) malformations we only used the diagnostic codes. We obtained migration dates and vital status from the Register of Populations and Population Changes, a registry that contains data on immigration and emigration since 1960. 


\section{Study population}

The study was restricted to men born in Sweden and who did not leave the country before attaining the age of 18 years. Men diagnosed with any malformation in the year prior to testicular cancer diagnosis were considered as unexposed to avoid any bias related to the completeness of information on malformation diagnoses in men being evaluated for testicular cancer. Age at diagnosis was defined according to first diagnosis date of the malformation.

A total of 7,552 TGCT cases were identified between 1964 and 2008. We excluded 707 males that were not born in Sweden, 30 males that emigrated from Sweden before age 18 years, 8 males that immigrated to Sweden after birth, and 214 males with missing information on county of birth. For each case we randomly selected five population controls from the population register, matching on year of birth and county of birth. Controls met the same inclusion criteria of cases and were alive and living in Sweden at the time of diagnosis of the corresponding case. The analysis included 6,593 individuals with TGCT, of whom 2,919 (44.3\%) had nonseminomas and 3,674 (55.7\%) had seminomas, and 32,955 controls.

The study was approved by the ethics committee of the Karolinska Institute. Informed consent was not required.

\section{Statistical analysis}

Conditional logistic regression was used to evaluate the association between each congenital malformation and TGCT cases and for cases classified by histologic type: seminomas and nonseminomas. Since the main and probably only determinants of completeness of the Hospital Discharge Registry, year of birth and county, were used as matching variables in our study, lack of detection of congenital malformations due to incomplete registration should not have introduced bias. Associations were also evaluated mutually adjusting for cryptorchidism, hypospadias/epispadias, inguinal hernia, urinary malformations and all 'other' genital malformations. We also evaluated models stratified by the median age at diagnosis for each congenital malformation. For cryptorchidism, we used age 13 instead of median age, based on evidence from a previous study that suggested that men who underwent orchiopexy after puberty might have a greater risk of testicular cancer than men who underwent orchiopexy before puberty (6).

In sensitivity analyses we excluded men born within 4 years prior to the start date of the hospital registry in each county to further account for potentially incomplete ascertainment of congenital malformations. We also restricted analyses to men born in 1973 onwards, as the Swedish Medical Birth Registry became complete in that year. In this subset of data we further evaluated potential confounding by maternal parity, birth weight, gestational age and maternal age. All analyses were performed with STATA/SE (STATA statistical software, version 11.1; StataCorp, College Station, TX). 


\section{RESULTS}

The median age at cancer diagnosis was 34 years. The median birth year for cases and controls was 1952 (interquartile range: 1945-1957). Birth characteristics for cases and controls born 1973 or later are reported in Table 1.

Cryptorchidism was associated with an increased risk of TGCT (OR: 3.18, 95\% CI: 2.50 4.04) (Table 2). Hypospadias (OR: 2.41, 95\% CI: 1.27-4.57), inguinal hernia (OR 1.37, 95\% CI: 1.11-1.68), and 'other' genital malformations (OR: 2.19, 95\% CI: 1.17-4.10) were also associated with an increased risk of TGCT. Mutual adjustment for cryptorchidism, hypospadias, inguinal hernia, urinary, and 'other' genital malformations did not appreciably change the ORs adjusting for year of birth and county of birth (cryptorchidism OR: 3.16, 95\% CI: 2.45, 3.96; hypospadias OR: 2.25, 95\% CI: 1.17-4.32; inguinal hernia OR: 1.30, 95\% CI: 1.06, 1.60; other genital malformations OR: 1.90, 95\% CI: 1.00, 3.63). For the other congenital malformations evaluated there was no evidence of an association with TGCT. Overall the combination of all congenital malformations except cryptorchidism, hypospadias/epispadias and inguinal hernia, was not associated with TGCT risk (OR: 1.16, 95\% CI: 0.93-1.44).

In analyses by histologic subtype, cryptorchidism (OR: 2.97, 95\% CI: 2.11-4.19), hypospadias/epispadias (OR: 6.11, 95\% CI: 2.53-14.75), inguinal hernia (OR: 1.45, 95\% CI: 1.11-1.90), other genital malformations (OR: 2.92, 95\% CI: 1.15-7.41) and the combined category of 'all malformations' (OR: 1.38, 95\% CI: 1.03-1.84) were associated with an increased risk of seminoma (Table 3). Cryptorchidism was associated with an increased risk of nonseminoma (OR: 3.40, 95\% CI: 2.43-4.76), whereas the other congenital malformations evaluated were not associated with an increased nonseminoma risk. For hypospadias, the magnitude of association across seminomas and nonseminomas was significantly heterogeneous ( $\mathrm{p}$-heterogeneity $<0.01$ ).

The OR associated with cryptorchidism increased from 2.76 (95\% CI: 2.09-3.65) for patients diagnosed before age 13 years (before puberty) to 4.96 (95\% CI: $3.06-8.04$ ) for patients diagnosed at age 13 years or later (Table 4; results from further analyses on whether patients had an orchiopexy before age 13 are reported in Supplementary Table 2). In contrast, earlier diagnoses of hypospadias, inguinal hernia and other genital malformations, were consistently associated with higher risk (Table 4). The risk of TGCT did not differ by age at diagnosis for the other (non-genital) malformations evaluated.

The results of sensitivity analyses which excluded men born within 4 years prior to the start date of the hospital register in each county, supported the relationship between cryptorchidism, hypospadias, inguinal hernia, and 'other' genital malformations and all TGCT (Supplemental Table 3). Analyses restricted to men born after 1973 also supported the associations (Supplemental Table 3). Further, the effect estimates restricted to men born after 1973 were not substantially different after adjustment for maternal age, birth weight and gestational duration (Supplemental Table 3). 


\section{DISCUSSION}

We observed that developmental urogenital abnormalities, in addition to cryptorchidism, resulted in an increased risk of TGCT. These findings support the hypothesis that prenatal exposures that affect proper genital development may be involved in the etiology of TGCT. These data are also consistent with animal data suggesting that prenatal exposures can disturb testicular development (summarized in (7)).

Cryptorchidism is a well-recognized risk factor for TGCT (overall and by histologic type) and our results were consistent with increased risk observed in many other studies (summarized in (4)). Fewer studies have evaluated hypospadias and risk of TGCT (3;5;810), but overall the findings support an increased risk. In analyses by histologic type, our data suggest that hypospadias is a risk factor for seminomas, a finding that should be interpreted with care and requires replication in additional large study populations. If the heterogeneous effect of hypospadias reflects a true etiologic difference, it may indicate that the associations between cryptorchidism, hypospadias and risk of testicular cancer may represent distinct etiologic pathways.

Inguinal hernia has been studied in numerous investigations with the majority of record or registry-based studies reporting increased risks and studies based on self-report also reporting increased risk, although less consistently (summarized in (4)). Congenital hernia is often associated with cryptorchidism, however we report increased risk of TGCT associated with inguinal hernia accounting for the cryptorchidism-TGCT association. We cannot rule out potential undiagnosed cryptorchidism among those with inguinal hernia, however, given that the median age at diagnosis of inguinal hernia was 24 , it is unlikely that a substantial proportion of individuals diagnosed with inguinal henia in our population have undiagnosed cryptorchidism.

The 'other genital malformations' category is a group of other specified anomalies of the genital organs. Results concerning other genital malformations are new as this group, to our knowledge, has not been analyzed before in association with testicular cancer. Additional research is needed to determine the potential biologic explanation for this association. We found a strong association between other genital malformations and testicular cancer that was not explained by cryptorchidism and/or hypospadias; it is possible that part of this association is explained by misclassification between these two conditions and other genital malformations. However, we do not believe that misclassification can explain the effect, not least because the OR associated with other genital malformations was of the same magnitude of that estimated for cryptorchidism or hypospadias.

We have previously assessed age at orchiopexy for cryptorchidism and found that treatment after age 13 is associated with higher risk for subsequent testicular cancer than is earlier orchiopexy (6). The present data are entirely consistent with that study, but it should be noted that these two studies to a large part share their study base. However, given that the number of exposed cases in this updated follow up is doubled and that we analyzed the data with a case-control approach, the current data provide further support that later orchiopexy is associated with an greater increased risk of cancer and thus confirms that testicular 
location is a critical factor in the development of germ cell tumors. Although an analysis of cohort data from Denmark did not support a benefit of surgery before puberty (12), findings from a recent meta-analysis further support that early surgery may lessen the increased risk of TGCT among cryptorchid men (13).

When the analyses were stratified by median age at diagnosis of the malformation, the associations with hypospadias and inguinal hernia seemed confined to or more pronounced among those diagnosed at younger ages. For hypospadias, early diagnosis of this malformation is likely a proxy for the grade of the malformation such that those diagnosed at earlier ages may have a more severe defect, and it does seem conceivable that the grade of birth defect could have a dose-response relation with risk of testicular cancer.

The associations of hypospadias and cryptorchidism with TGCT have been often discussed within the framework of the concept of a Testicular Dysgenesis Syndrome, which postulated that these conditions, as well as poor semen quality, resulted from disrupted embryonal programming and gonadal development caused by the same exposures, possibly of environmental origin (14). As the risk factors for all of the conditions remain poorly defined, it is not yet possible to determine whether they share any in common (3). As the current study is aimed at estimating the separate (conditionally independent) associations between the congenital malformations and the risk of TGCT, the study is unable to provide information on whether the conditions resulted from common risk factors.

The strengths of our study include the use of nationwide population-based design, long-term follow-up and large sample size given the use of the Swedish registry data. The categorization of malformations diagnosed one year before testicular cancer diagnosis as unexposed limited the potential impact of detection bias. A limitation of our study was the completeness of the Hospital Discharge Register over time. Calendar year and geographic region are the only determinants of completeness of the Hospital Discharge Register and those variables were matching factors in our study to account for the incompleteness of exposure data over time. In sensitivity analyses excluding men born within 4 years prior to the start date of the hospital registry in each county and restricting to men born after 1973 supported the observed associations.

In conclusion, this large, population-based study suggests that genital malformations are associated with increased risk of TGCT, and specifically that hypospadias and inguinal hernia in addition to cryptorchidism are risk factors for TGCT; further supporting the hypothesis that prenatal exposure(s) related to proper genital development are related to this tumor.

\section{Supplementary Material}

Refer to Web version on PubMed Central for supplementary material.

\section{Acknowledgments}

We thank Milena Maule for her contribution to the data analysis. This work was supported by grants from the Swedish Cancer Society (4952-B04-01XAA); the Compagnia SanPaolo Foundation (to Dr. Richiardi); the Swedish 
Cancer Society (to Dr. Akre); and the Intramural Research Program on the National Cancer Institute (Drs. Trabert and McGlynn).

\section{Abbreviations}

$\begin{array}{ll}\text { CI } & \text { confidence interval } \\ \text { OR } & \text { odds ratio } \\ \text { TGCT } & \text { testicular germ cell tumors }\end{array}$

\section{References}

1. Chia VM, Quraishi SM, Devesa SS, Purdue MP, Cook MB, McGlynn KA. International trends in the incidence of testicular cancer, 1973-2002. Cancer Epidemiol Biomarkers Prev. 2010; 19:11511159. [PubMed: 20447912]

2. Rajpert-De ME, Hoei-Hansen CE. From gonocytes to testicular cancer: the role of impaired gonadal development. Ann N Y Acad Sci. 2007; 1120:168-180. [PubMed: 18184914]

3. Akre O, Richiardi L. Does a testicular dysgenesis syndrome exist? Hum Reprod. 2009; 24:20532060. [PubMed: 19443456]

4. Cook MB, Akre O, Forman D, Madigan MP, Richiardi L, McGlynn KA. A systematic review and meta-analysis of perinatal variables in relation to the risk of testicular cancer--experiences of the son. Int J Epidemiol. 2010; 39:1605-1618. [PubMed: 20660640]

5. Schnack TH, Poulsen G, Myrup C, Wohlfahrt J, Melbye M. Familial coaggregation of cryptorchidism, hypospadias, and testicular germ cell cancer: a nationwide cohort study. J Natl Cancer Inst. 2010; 102:187-192. [PubMed: 20026812]

6. Pettersson A, Richiardi L, Nordenskjold A, Kaijser M, Akre O. Age at surgery for undescended testis and risk of testicular cancer. N Engl J Med. 2007; 356:1835-1841. [PubMed: 17476009]

7. Sharpe RM. Pathways of endocrine disruption during male sexual differentiation and masculinization. Best Pract Res Clin Endocrinol Metab. 2006; 20:91-110. [PubMed: 16522522]

8. Prener A, Engholm G, Jensen OM. Genital anomalies and risk for testicular cancer in Danish men. Epidemiology. 1996; 7:14-719. [PubMed: 8664395]

9. Aetiology of testicular cancer: association with congenital abnormalities, age at puberty, infertility, and exercise. United Kingdom Testicular Cancer Study Group. BMJ. 1994; 308:1393-1399. [PubMed: 7912596]

10. Moller H, Prener A, Skakkebaek NE. Testicular cancer, cryptorchidism, inguinal hernia, testicular atrophy, and genital malformations: case-control studies in Denmark. Cancer Causes Control. 1996; 7:264-274. [PubMed: 8740739]

11. Mylchreest E, Wallace DG, Cattley RC, Foster PM. Dose-dependent alterations in androgenregulated male reproductive development in rats exposed to Di(n-butyl) phthalate during late gestation. Toxicol Sci. 2000; 55:143-151. [PubMed: 10788569]

12. Myrup C, Schnack TH, Wohlfahrt J. Correction of cryptorchidism and testicular cancer. N Engl J Med. 2007; 357:825-827. [PubMed: 17715418]

13. Banks K, Tuazon E, Berhane K, et al. Cryptorchidism and testicular germ cell tumors: comprehensive meta-analysis reveals that association between these conditions diminished over time and is modified by clinical characteristics. Front Endocrinol (Lausanne). 2012; 3:1-11. (article 182). [PubMed: 22649402]

14. Skakkebaek NE, Rajpert-De ME, Main KM. Testicular dysgenesis syndrome: an increasingly common developmental disorder with environmental aspects. Hum Reprod. 2001; 16:972-978. [PubMed: 11331648] 


\section{Novelty and impact}

While cryptorchidism is a known risk factor for testicular germ cell tumors (TGCT), few studies have evaluated the association between other birth defects and risk of TGCT. Utilizing large population-based registries in Sweden we evaluated this important question. Our results demonstrate that hypospadias, inguinal hernia and other genital malformations are associated with an increased risk of TGCT. These findings highlight the importance of prenatal exposures related to proper genital development in the etiology of TGCT. 


\section{Table 1}

Demographic characteristics and birth registry information for testicular germ cell tumors and matched controls, Swedish Cancer Registry, 1964-2008. ${ }^{*}$

\begin{tabular}{|c|c|c|c|c|}
\hline & \multicolumn{2}{|c|}{$\begin{array}{c}\text { Case } \\
(\mathrm{n}=6,593)\end{array}$} & \multicolumn{2}{|c|}{$\begin{array}{c}\text { Controls } \\
(\mathbf{n}=\mathbf{3 2 , 9 5 5})\end{array}$} \\
\hline & $\mathbf{n}$ & $\%$ & $\mathbf{n}$ & $\%$ \\
\hline \multicolumn{5}{|c|}{ Age at cancer diagnosis, years } \\
\hline $15-19$ & 241 & 3.7 & & \\
\hline $20-29$ & 2166 & 32.8 & & \\
\hline 30-39 & 2281 & 34.6 & & \\
\hline $40-49$ & 1222 & 18.5 & & \\
\hline $50-59$ & 514 & 7.8 & & \\
\hline $60-65$ & 169 & 2.6 & & \\
\hline \multicolumn{5}{|l|}{ Year at cancer diagnosis } \\
\hline 1964-1972 & 636 & 9.6 & & \\
\hline 1973-2008 & 5957 & 90.4 & & \\
\hline \multicolumn{5}{|l|}{ Year of birth } \\
\hline$<1964$ & 4350 & 66.0 & 21750 & 66.0 \\
\hline 1964-1969 & 956 & 14.5 & 4780 & 14.5 \\
\hline 1970-1974 & 623 & 9.4 & 3110 & 9.4 \\
\hline 1975-1979 & 405 & 6.1 & 2025 & 6.1 \\
\hline 1980-1984 & 202 & 3.1 & 1010 & 3.1 \\
\hline$\geq 1985$ & 57 & 0.9 & 280 & 0.9 \\
\hline Data for 1973-2008 only & Mean & SD & Mean & SD \\
\hline Birthweight, grams & 3525.6 & 574.2 & 3562.8 & 568.0 \\
\hline Maternal age, years & 26.9 & 4.9 & 26.9 & 5.0 \\
\hline Gestational age, weeks & 39.4 & 2.0 & 39.6 & 1.9 \\
\hline Maternal parity status & $\mathrm{n}$ & $\%$ & $\mathrm{n}$ & $\%$ \\
\hline Nulliparous & 388 & 43.7 & 1875 & 42.7 \\
\hline Parous & 499 & 56.3 & 2514 & 57.3 \\
\hline
\end{tabular}


Table 2

Associations between congenital malformation and testicular germ cell tumors, Swedish Cancer Registry, 1964-2008. *

\begin{tabular}{lcccc}
\hline & $\begin{array}{c}\text { Cases } \\
\mathbf{n}(\boldsymbol{\%})\end{array}$ & $\begin{array}{c}\text { Controls } \\
\mathbf{n}(\boldsymbol{\%})\end{array}$ & $\mathbf{O R}^{\dagger}$ & $(\mathbf{9 5 \%} \mathbf{C I})$ \\
\cline { 2 - 5 } Cryptorchidism & $113(1.71)$ & $181(0.55)$ & 3.18 & $(2.50-4.04)$ \\
Hypospadias/Epispadias & $14(0.21)$ & $29(0.09)$ & 2.41 & $(1.27-4.57)$ \\
Inguinal hernia & $118(1.79)$ & $434(1.32)$ & 1.37 & $(1.11-1.68)$ \\
CNS & $6(0.09)$ & $28(0.08)$ & 1.07 & $(0.44-2.59)$ \\
Cleft lip & $6(0.09)$ & $30(0.09)$ & 1.00 & $(0.42-2.40)$ \\
Digestive tract & $6(0.09)$ & $28(0.08)$ & 1.07 & $(0.44-2.60)$ \\
Circulatory & $13(0.20)$ & $44(0.13)$ & 1.48 & $(0.80-2.76)$ \\
Musculo-skeletal & $18(0.27)$ & $105(0.32)$ & 0.86 & $(0.52-1.41)$ \\
Ear & $18(0.27)$ & $78(0.24)$ & 1.15 & $(0.69-1.93)$ \\
Eye & $4(0.06)$ & $14(0.04)$ & 1.43 & $(0.47-4.34)$ \\
Urinary system & $11(0.17)$ & $45(0.14)$ & 1.22 & $(0.63-2.36)$ \\
Other genital ${ }^{\ddagger}$ & $14(0.21)$ & $32(0.10)$ & 2.19 & $(1.17-4.10)$ \\
Other (non-genital) & $14(0.21)$ & $64(0.19)$ & 1.09 & $(0.61-1.95)$ \\
All malformations ${ }^{\ddagger}$ & $104(1.58)$ & $451(1.37)$ & 1.16 & $(0.93-1.44)$ \\
\hline * & & &
\end{tabular}




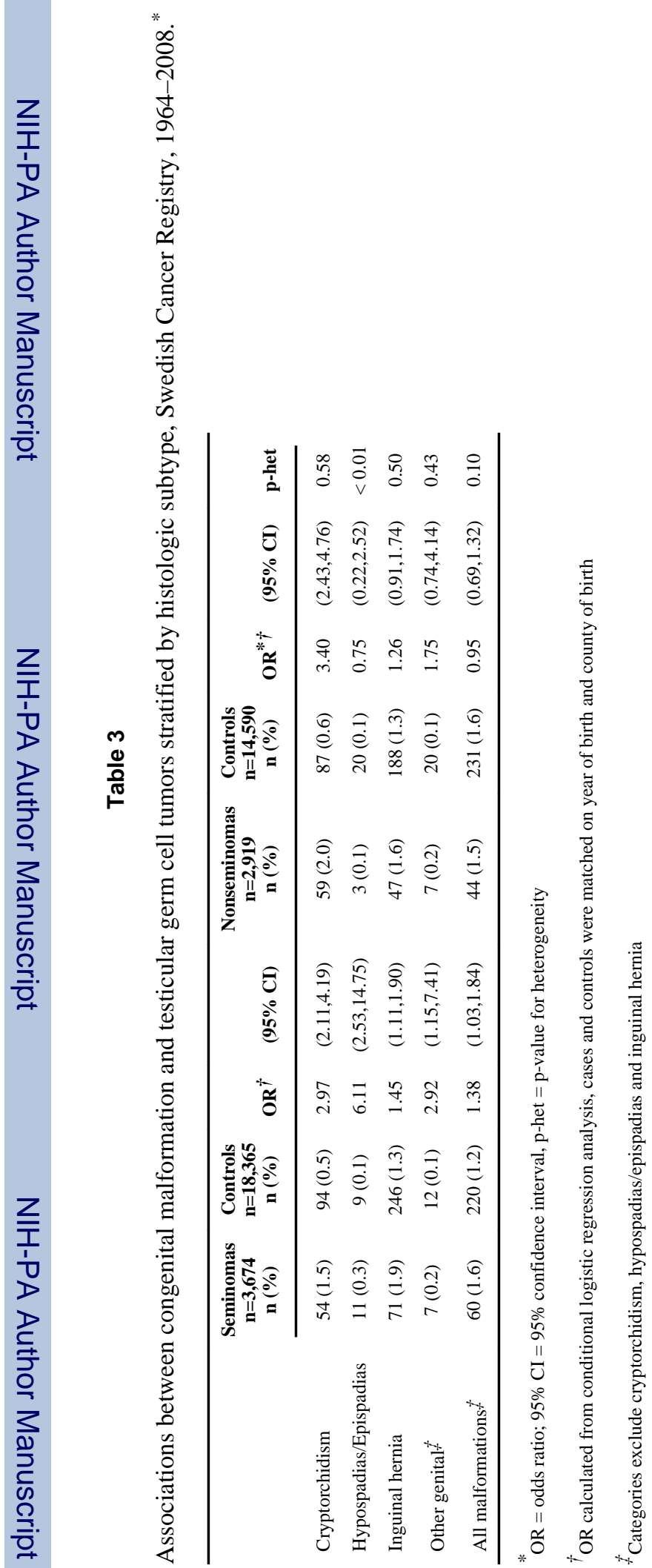


